\title{
Characterization of the immune defense related tissues, cells, and genes in amphioxus
}

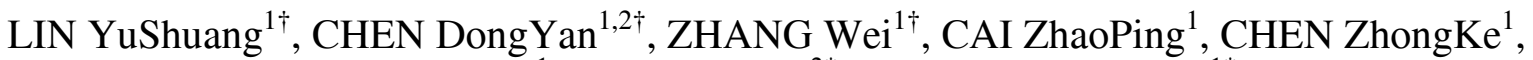 \\ ZHANG Ning ${ }^{1}$, MAO Bing Yu ${ }^{3 *} \&$ ZHANG HongWei ${ }^{1 *}$ \\ ${ }^{1}$ Life Science College, Shandong University, Key Laboratory of Experimental Teratology of Ministry of Education of China, Shandong \\ Provincial Key Laboratory of Animal Cells and Developmental Biology, Jinan 250100, China; \\ ${ }^{2}$ Medical School, Nankai University, Tianjin 300071, China; \\ ${ }^{3}$ Kunming Institute of Zoology, Chinese Academy of Sciences, Kunming 650223, China
}

Received September 10, 2011; accepted November 10, 2011

\begin{abstract}
Amphioxus is an important animal model for phylogenetic analysis, including comparative immunology. Exploring the immune system in amphioxus contributes to our understanding of the origin and evolution of the vertebrate immune system. We investigated the amphioxus immune system using ultrastructural examination and in situ hybridization. The expression patterns of TLR1 (toll-like receptor 1), ClQ (complement component 1, q subcomponent), ECSIT (evolutionarily conserved signaling intermediate in Toll pathways), SoxC, DDAHa (Dimethylarginine dimethylaminohydrolase a), and NOS (nitric oxide synthase) show that these genes play key roles in amphioxus immunity. Our results suggest that the epidermis and alimentary canal epithelium may play important roles in immune defense, while macrophages located in the coelom and so-called lymph spaces may also be crucial immune cells.
\end{abstract}

amphioxus, epidermis, alimentary canal, macrophages, immunity, evolution

Citation: Liu Y S, Chen D Y, Zhang W, et al. Characterization of the immune defense related tissues, cells, and genes in amphioxus. Sci China Life Sci, 2011, 54: 999-1004, doi: 10.1007/s11427-011-4237-z

Amphioxus, a cephalochordate representing the invertebrate-to-vertebrate transition, is an important experimental animal model for phylogenetic analysis, including comparative immunology. However, the amphioxus immune system remains largely unexplored.

Amphioxus possesses the basic vertebrate structures, including a notochord, dorsal neural cord, intramuscular somites, and pharynx region with gill slits. Similar to other chordates, the exterior covering of the amphioxus body is skin, which can provide protection against the invasion of microorganisms and play an important role in innate im-

$\dagger$ Contributed equally to this work

*Corresponding author (email: mao@mail.kiz.ac.cn; zhw@sdu.edu.cn) mune responses. Amphioxus skin consists of a single layer of columnar epithelium or pseudostratified epithelium covered by a thin film of keratose cuticles. Distinct ultrastructural characteristics of endocytosis in amphioxus epidermal cells at the 24-h larva stage have been observed [1]. A key amphioxus immune organ is the alimentary canal just after the mouth, which can be divided into the pharynx region and the intestine. The intestinal epithelium in amphioxus plays an essential role in amphioxus immune defense against LPS challenge [2].

The amphioxus body possesses many cavities, including the coelom and so-called lymph spaces. The coelom consists of the following sections, the suprapharyngeal, subchordal, branchial, perienteric, perigenital, endostyle, and 
metapleure. Lymph spaces are considered to be discontinuous with the coelom [3]. Important immune defense related cells include macrophages in the coelom and lymph spaces, and a small number of granulocytes in blood vessels, which have been confirmed by transmission and scanning electron microscopy [4].

Amphioxus has always been considered to lack an adaptive immune system. However, using molecular methods, Cannon et al. [5] discovered the $\mathrm{V}$ region-containing chitin binding proteins (VCBPs), which represent a core feature of the adaptive immune receptors in amphioxus. Genome sequencing of Branchiostoma floridae revealed a series of homologous chordate genes and gene families involved in immune defense [6-10]. Although some studies have been published, more research is required to better understand the origin of immune recognition and immune defense in amphioxus. TLRs play an important function in triggering the innate immune response and ECSIT is an evolutionarily conserved intermediate in the Toll/IL-1 signal transduction pathway. C1Q is the recognition protein of the classical complement pathway and is an important link between the innate and acquired immune system. Sox 4 , a member of the SoxC family, is expressed in $\mathrm{T}$ and pre-B lymphocytes in the murine thymus [11]. DDAH and NOS regulate the synthesis of NO, which serves as a primary immune activator and a signaling molecule with multiple roles in vertebrates [12-14]. Therefore, we first analyzed the expression pattern of genes involved in vertebrate immunity, such as TLRI, ECSIT, CIQ, SoxC, DDAHa, and NOS in amphioxus. At the same time, we investigated the ultrastructural characteristics of the epidermis and epithelium of the gill and intestine. This expression analysis and the ultrastructural analysis may provide more clues and a better understanding of the evolution of the vertebrate adaptive immune system.

\section{Materials and methods \\ 1.1 Amphioxus collection}

Adult amphioxus (Branchiostoma belcheri) were caught in the Yellow Sea near Qingdao, China and the South Sea near Beihai, Guangxi, and spawned in the laboratory [15]. Synchronously developing embryos and larvae were cultured and collected at different development stages.

Embryos and larvae were fixed for whole-mount in situ hybridization in $4 \%$ paraformaldehyde in $0.1 \mathrm{~mol} \mathrm{~L}^{-1}$ 3-(N-morpholino)-propanesulfuric acid buffer with $1 \mathrm{mmol}$ $\mathrm{L}^{-1}$ ethyleneglycotetraacetic acid, and $0.5 \mathrm{~mol} \mathrm{~L}{ }^{-1} \mathrm{NaCl}(\mathrm{pH}$ 7.5 ) at room temperature for $1 \mathrm{~h}$ and stored in $70 \%$ ethanol at $-20^{\circ} \mathrm{C}$.

\subsection{Ultrastructural studies}

Adult and larval specimens for transmission electron microscopy (TEM) were cut into 1-2 mm blocks and fixed in
$4 \%$ glutaraldehyde in $0.2 \mathrm{~mol} \mathrm{~L}^{-1}$ sodium cacodylate, $\mathrm{pH}$ 7.4, for $2 \mathrm{~h}$ at room temperature. Larvae were cultured for 30 min in sea water, with a drop of Chinese ink, before fixation to detect endocytosis using carbon particles of cells under TEM.

Specimens were postfixed in $1 \%$ osmium tetroxide for 1 $\mathrm{h}$, followed by dehydration in a graded ethanol series and embedded in Epon 812. Semi-thin-sections $(1 \mu \mathrm{m})$ were prepared for primary observation, followed by preparation of a series of ultrathin sections $(0.05 \mu \mathrm{m})$. After staining with uranyl acetate and lead citrate, ultrathin sections were observed and photos taken under a JEM-200CX electron microscopy.

\subsection{Cloning of amphioxus TLR, ECSIT, C1Q, SoxC, $D D A H a$, and NOS genes}

To generate in situ hybridization probes, PCR was performed with the following primers using sequences from NCBI or JGI: TLR1 (DQ400125: F, 5'-CACAGCATCGCAACAATCCC-3'; R, 5'-CGCAGCCTCCTCCAGAACAG-3'); ECSIT (XM_002595374: F, 5'-GAGGCCATGTGGAGTTTATCTA-3'; R, 5'-GGGGAACCAGTACAGGATACG-3'); C1Q (XM_002586916: F, 5'-TACAGGTCCACAAGGCCCA-3'; R, 5'-AGAAGAAACCCGGTGAAG-3'); DDAHa (DQ860841: F, 5'-ACTGGTGGAGGTTTGATGTC-3'; R， 5'-CCGCAAGGAACTCGGAATGT-3'); NOS (AF396968: F, 5'-AGGAGGACGCCTGGTTGGTA-3'; R, 5'-CGGGTGCCTCATGTGAAGTAA-3') and SoxC (FJ176301: F， 5'-CGTTTATGGTCTGGTCTCAGATC-3'; R, 5'-GCTTTCTTCACCTTTTTCC-3'). All PCR products were cloned, sequenced, and used as templates for making digoxigenin (DIG) labeled probes.

\subsection{Section in situ hybridizations}

DIG-labeled sense and antisense RNA probes were prepared according to manufacturer's instructions (Dig RNA Labeling Mix, Roche, USA; SP6 or T7 RNA Polymerases, Promega, USA). Adult transverse sections $(8 \mu \mathrm{m})$ were mounted onto slides (ThermoFisher, USA). After deparaffinization, sections were rehydrated in gradient ethanol, immersed in $0.3 \%$ Triton $\mathrm{X}-100$ solution for $15 \mathrm{~min}$ at room temperature and in proteinase $\mathrm{K}$ solution for $20 \mathrm{~min}$ at $37^{\circ} \mathrm{C}$. Sections were then incubated with $4 \%$ paraformaldehyde/PBS for $5 \mathrm{~min}$ at $4^{\circ} \mathrm{C}$. After washing with PBS and 0.1 mol L $\mathrm{L}^{-1}$ triethanolamine buffer containing $0.25 \%$ acetic anhydride, the slides were incubated with prehybridization solution for $2 \mathrm{~h}$ at $37^{\circ} \mathrm{C}$. Sense and antisense probes were added at a concentration of $1 \mu \mathrm{g} \mathrm{mL} \mathrm{m}^{-1}$ and hybridized overnight at $50^{\circ} \mathrm{C}$. After high stringency washing, sections were incubated with an alkaline phosphatase-conjugated-antidigoxigenin antibody, and NBT/BCIP substrate, and monitored for a color reaction. 
1.5 Injection of Staphylococcus aureus Rosenbach induces different expression patterns of TLR, ECSIT, C1Q, SoxC, DDAHa and NOS in amphioxus

Amphioxus subjected to celiac injections with Staphylococcus aureus Rosenbach in phosphate buffered solution (PBS) (experimental group) and germ-free PBS (control group) were processed as follows. After culturing the injected amphioxus in sea water for $3 \mathrm{~d}$, RNA was isolated from the alimentary canal, RT-PCR performed with the above primers (in materials and methods 1.3) and $\beta$-actin primers (F, 5'-GGAAGAGAGACTCGGGGC-3'; R, 5'-CTCACAGAGCGTGGCTACAG-3'), to determine expression patterns of TLR, ECSIT, CIQ, SoxC, DDAHa, and NOS.

\section{Results}

2.1 Ultrastructural studies on epidermis, gill epithelium and alimentary canal as well as macrophages in larval and adult amphioxus

Epidermal cells of 24 to 72-h amphioxus larvae were examined by TEM after culturing for $30 \mathrm{~min}$ in seawater containing ink. As shown in Figure 1, the monolayer epidermal cells possessed numerous vesicles containing different electron-dense materials in the cytoplasm. Several vesicles with tracer carbon particles were in apical cytoplasm and the endocytosing vesicles on the cytoplasmic membrane could be observed in epidermal cells (Figure 1A and B). The nucleus is located close to the basal surface of the cell (Figure $1 \mathrm{~A}$ and $\mathrm{B})$. Between adjacent epidermal cells, typical septate junctions were detected in the apical area, with no tight junction (Figure 1C). Tracing carbon particles were also found in the intercellular spaces (Figure 1C), suggesting that larval epidermal cells are capable of endocytosis.

The ultrastructural observations were also performed on the differentiating branchial cells and epithelial cells of the alimentary canal, from 48 to 72 -h amphioxus larva. Prominent characteristics of larval branchial epithelial cells were numerous cilia orientated to pharyngeal lumen on the apical surface and developed Golgi apparatus in the cytoplasm (Figure 1D). The epithelial cells of a nascent alimentary canal appeared as short columns. Their primary distinguishing feature was the presence of numerous vesicles with low electron-dense substances. The cytoplasm usually contained numerous rounded or elliptic mitochondria, endoplasmic reticulum, and abundant free ribosomes (Figure 1E). Strikingly, only zonula adherens rather than tight or septate junctions were found between adjacent epithelial cells of the alimentary canal. A migrating cell was occasionally observed in the epithelium of the alimentary canal of amphioxus larva with variable shape and more electron-dense nucleus in cytoplasm, and the shape of the cell and nucleus might suggest the migrating direction (Figure 1F). The migrating cell might be the precursor of mononuclear free cells, granulocytes, or macrophages. The macrophages in the perigenital coelom of larvae and adults exhibited irregular shapes and many cellular protrusions. Numerous vesicles, multivesicular bodies, lysosomal granules and phagosomes with different electron-dense substance were observed in the cytoplasm of macrophages (Figure 1G).

\subsection{Expression patterns of immune defense related genes in epidermis, gill epithelium, intestinal epithelium, and macrophages}

Several homologs of vertebrate immune system genes were detected in the epidermis, gill and intestine epithelium, as

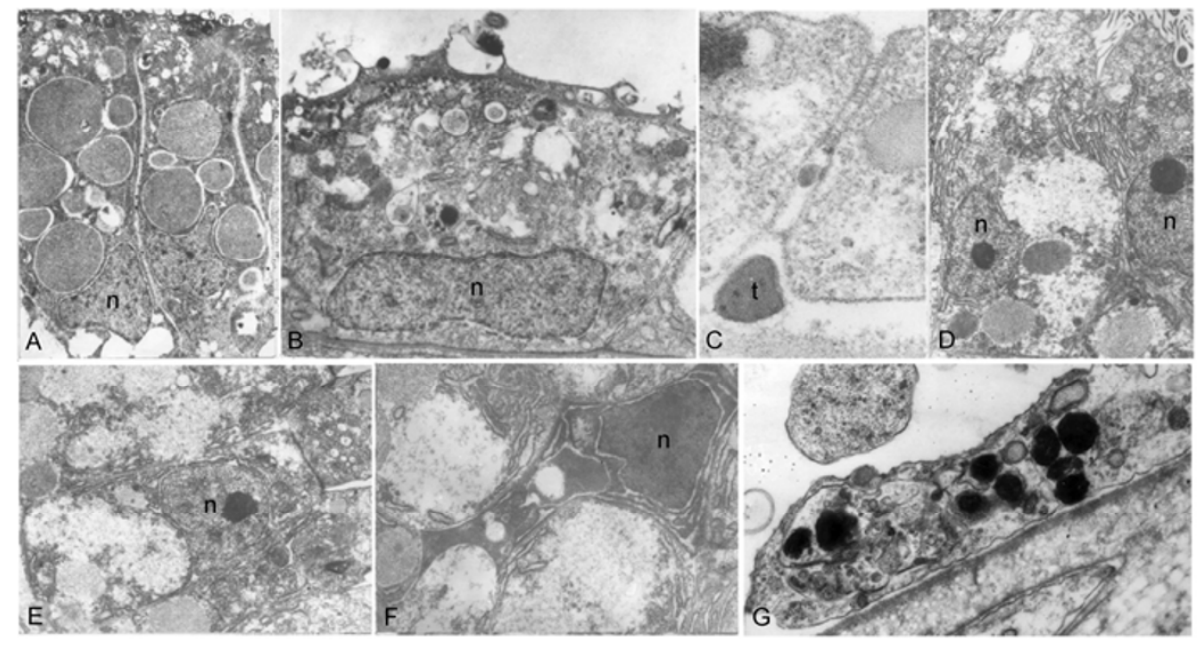

Figure 1 Transmission electron micrographs of epidermal cells, branchial cells, epithelium of the alimentary canal and macrophages in amphioxus. A and B, Epidermal cells in 24-h and 48-h larva, respectively. The vesicles with tracer carbon particles in the apical cytoplasm and endocytosing vesicles on the cytoplasmic membrane can be observed. C, At the 36 to 48 -h larva stage, carbon tracer particles supplied in cultured seawater are observed in the intercellular space. D, The branchial epithelial cells of 48-h larva. E, The epithelial cells in the differentiating alimentary canal of 48-h larva. F, A migrating cell in the epithelium of alimentary canal in 72-h larva. G, A macrophage is observed in the perigenital coelom of adult amphioxus. n, nucleus; t, tracer carbon particles. Magnification: A, ×12300; B, ×155000; C-E, ×11000; F, ×8700; G, ×8500. 
well as macrophages. These genes were TLRI [16], SoxC [8], and DDAHa [17], respectively. Moreover, we also detected expression of ECSIT, ClQ and NOS homologs, whose amino-acid sequences were over $40 \%$ identical to human.

TLRI was clearly expressed in the epidermal cells covering the body (Figure 2A), in the cytoplasm of intestinal epithelial cells (Figure 2B), and in all ciliary epithelial cells of the branchial lamellas (Figure 2C). The expression of TLRI was limited to macrophages located in the coelom and lymphoid cavities (Figure 2B-E).

The expression patterns of $C 1 Q, E C S I T$, and SoxC were similar to TLRI in the pharyngeal region, which were observed in all ciliary epithelial cells of the branchial lamella, the wall of the septal coelom, as well as in coelomic macrophages (Figure $3 \mathrm{~A}-\mathrm{C}$ ). Expression of DDAHa and NOS was also detected in the branchial cells except for the lateral ciliary epithelial cells (Figure 3D and E). Similar to TLR1, all genes were expressed in all epithelial cells of the intestine and the macrophages in the perienteric coelom (Figure
$3 \mathrm{~F}-\mathrm{J})$. Similar to TLR1, DDAHa is expressed in all epidermal cells while expression of the other genes appeared mainly in the epidermal cells at the metapleural fold region (Figure 3K-O).

\subsection{Staphylococcus aureus Rosenbach alters expression of TLR, ECSIT, ClQ, SoxC, DDAHa, and NOS in am- phioxus}

RT-PCR results show that after amphioxus was injected with Staphylococcus aureus Rosenbach, the ECSIT, TLR, and $C 1 Q$ were up-regulated. The $D D A H a$ was up-regulated slightly in amphioxus. The expression of NOS did not change. It was interesting to find that SoxC was down-regulated after injection of Staphylococcus aureus Rosenbach (Table 1).

\section{Discussion}

One main function of skin is to protect an organism against
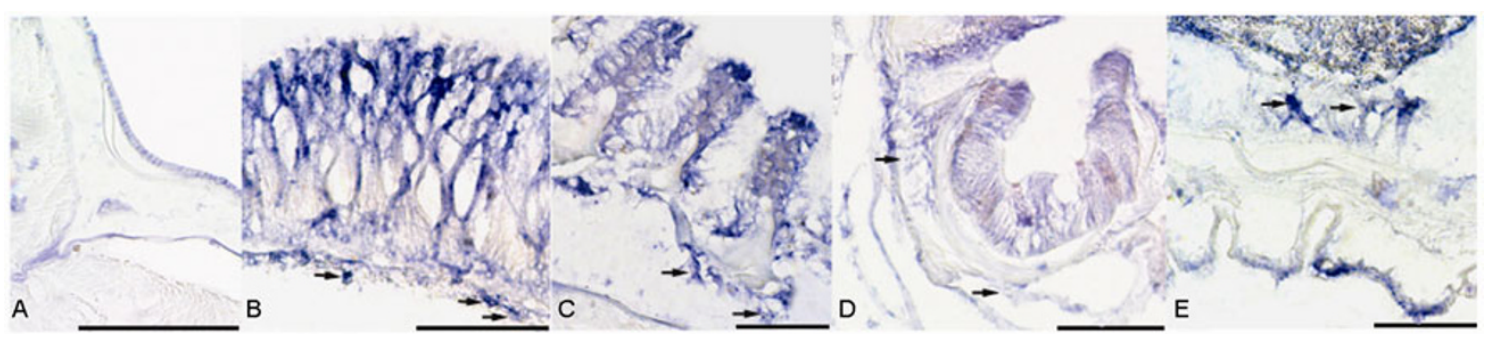

Figure 2 The expression of TLR1 is detected in epidermis, gill epithelium and the intestinal epithelium as well as macrophages by in situ hybridization. A, Expression of TLRI in epidermal cells near the notochord. B, Expression of TLRI in intestinal epithelial cells and macrophages (arrows) in perienteric coelom. C, TLR1 expression in gill epithelium and macrophages (arrows) in the branchial coelom. D, The expression of TLRI is detected in endostyle epithelial cells and macrophages (arrows) in lymphoid cavities. E, Expression of TLR1 is also observed in the epithelial cells of metapleure and perigenital coelomic macrophages (arrows). Scale bars, $50 \mu \mathrm{m}$.

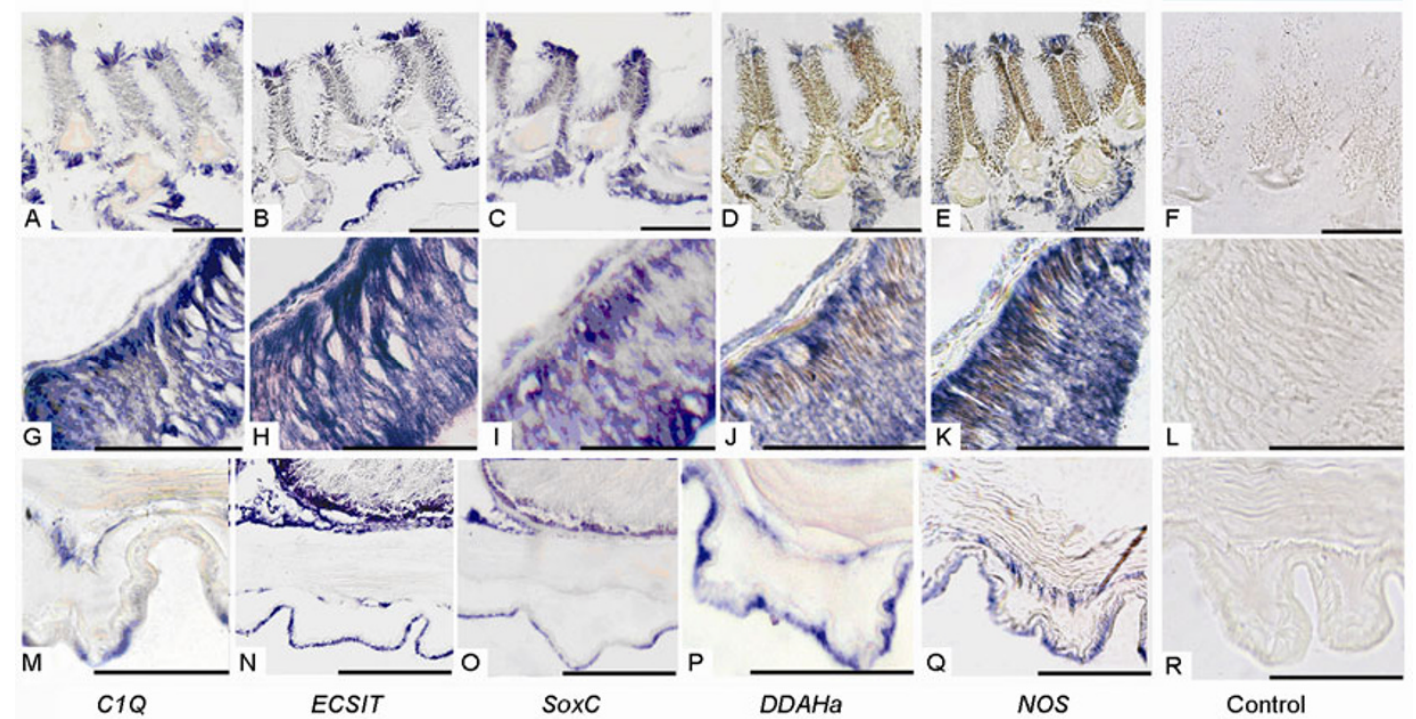

Figure 3 The expression pattern of CIQ, ECSIT, SoxC, DDAHa, and NOS of amphioxus gill epithelium and branchial coelom macrophages (A-E); the intestinal epithelium and perienteric coelom macrophages $(\mathrm{G}-\mathrm{K})$; the metapleural fold epidermis and metapleure coelom macrophages (M-Q) by in situ hybridization. F, L, and R are controls using sense DDAHa RNA probes. Scale bars, $50 \mu \mathrm{m}$ for A-L and $25 \mu \mathrm{m}$ for M-R. 
external pathogens. Early studies found that the amphioxus epidermis could secret lectins and other antibacterial peptides [18]. Our previous report [1] and the TEM data presented here show that epidermal cells of amphioxus larva older than $24 \mathrm{~h}$ possess typical morphologic characteristics, such as endocytic vesicles on the cell membrane and endocytosis particles in the apical cytoplasm. Furthermore, tracing carbon particles were observed in the intercellular spaces of epidermal cells. These data indicate that amphioxus larval epidermal cells are capable of endocytosis. The less electron-dense vesicles in the cytoplasm were more likely involved in lectin and antibacterial peptide secretion. Additionally, the in situ hybridization results suggest that expression of TLRI in epidermis is similar to TLRs expression in human keratinocytes, where a functional TLR is expressed and that contains an antimicrobial defense barrier [19]. We also demonstrate the expression of other potential immune defense vertebrate homologs, including $C 1 Q$, ECSIT, SoxC, DDAHa, and NOS. This is consistent with the suggestion that epidermis performs an important role in the immune defense of amphioxus.

The immune system is composed of the innate and adaptive immune systems. TLRs play a crucial role in the recognition and induction of the immune response. Immunocytes, such as macrophages, dendritic cells, and B lymphocytes recognize pathogens via TLRs. The stimulation of TLRs via microbial invasion activates the innate immune response. In some cases, activation of antigen-presenting cells (APCs) via TLRs up-regulates nitric oxide and induces the adaptive immune response [20]. ECSIT is a regulator involved in both BMP and TLR signaling. C1Q, a recognition protein in the classical complement system, is involved in both innate and acquired immunity. SoxC, which plays a role in lymphocyte maturation, is expressed in tissues where macro- phages exist [11]. DDAHa and NOS are involved in the regulation of endogenous NO synthesis [21], and NO is active in both acquired and innate immunity. Following celiac injection with $S$. aureus, we observed up-regulation of ECSIT, TLR, and CIQ, which suggests these genes play an important role in immune defense. The little changed expression of $\mathrm{DDAHa}$ implies that stimulation by $\mathrm{S}$. aureus may have no direct influence on NOS expression. The down-regulation of SoxC indicates that SoxC might have a different role in immune defense. We found that similar to $T L R 1$, expression of $C 1 Q, E C S I T$, SoxC, DDAHa, and NOS appear in gill and intestinal epithelial, suggesting that in addition to the epidermis, the entire alimentary canal has an immune related role in amphioxus. Previous studies have indicated that one of VCBP genes, $V C B P 1$, is expressed selectively in the alimentary canal [5]. TLRI is also expressed in amphioxus gut and gill, which implies that the alimentary canal in amphioxus is likely to be the major immune system tissue. It is interesting to find potential precursors to free mononuclear cells, granulocytes or macrophages, in the differentiating epithelium of the alimentary canal in larva. It is worth noting that potential homologs of TLR1, C1Q, ECSIT, SoxC, DDAHa, and NOS are also expressed in amphioxus macrophages, suggesting a crucial immune function for macrophages in this animal.

In amphioxus, some lymphoid related genes, such as the Ikaros-like gene and the Bam32 gene (B lymphocyte adaptor molecule of $32 \mathrm{kD}$ ) [22] are expressed in the metapleural fold and intestine (Table 2). Other immune defense related genes show similar expression patterns in immune related tissues (epidermis and the alimentary canal) and cells (macrophage), which suggests that all these genes play a role in immune defense.

Table 1 RT-PCR detection of ECSIT, TLR, CIQ, DDAHa, SoxC, and NOS expression ${ }^{\text {a) }}$

\begin{tabular}{|c|c|c|c|c|c|c|}
\hline & ECSIT & $T L R 1$ & $C 1 Q$ & $D D A H a$ & $N O S$ & SoxC \\
\hline $\begin{array}{l}\text { Expression pattern changes after } \\
\text { injection }\end{array}$ & $\uparrow \uparrow \uparrow$ & $\uparrow \uparrow$ & $\uparrow \uparrow$ & $\uparrow$ & - & $\downarrow$ \\
\hline
\end{tabular}

a) The number of ' $\uparrow$ ' indicates the level of up-regulation; '-' indicates no significant change in expression; ' $\downarrow$ ' indicates the level of down-regulation.

Table 2 Comparison of the expression patterns of homologous immune defense genes

\begin{tabular}{ccccc}
\hline & Macrophages in coelom & Epidermal cells of metapleural fold & Pharynx epithelium & Gut epithelium \\
\hline TLR & + & + & + & + \\
ECSIT & + & + & + & + \\
C1Q & + & + & + & + \\
SoxC & + & + & + & + \\
DDAHa & + & + & + & + \\
NOS & + & + & + & + \\
Thymosin $\beta 4[23]$ & & & + & + \\
Ikaros-like gene [22] & + & + & & + \\
Bam32 [22] & & & & \\
VCBP [5] & & & & + \\
\hline
\end{tabular}


In summary, we report the ultrastructural characteristics of amphioxus larvae epidermis, gill, and intestine epithelium. Our results indicate that the epidermis and the alimentary canal play important roles in immune defense, while macrophages in the coelom and so-called lymph spaces may be crucial immune cells. Moreover, in situ expression patterns of TLR1, CIQ, ECSIT, SoxC, DDAHa, and NOS suggest that they play a role in an immune response. Our studies should facilitate understanding of the amphioxus immune system and evolution of the primordial adaptive immune system.

We thank Prof. Chen JunYuan, Nanjing University, and Prof. Zhang PeiJun, Institute of Oceanology, Chinese Academy of Sciences for assistance and providing amphioxus. This work was supported by the National Natural Science Foundation of China (Grant Nos. 30570967, 30671072 and 30700434), Natural Science Foundation of Shandong Province (Grant No. Y2007D20), and Ministry of Science and Technology of China (Grant Nos. 2007CB815800 and 2007CB947100).

1 Mao B Y, Sun X Y, Zhang H W, et al. Morphological and functional studies on the epidermal cells of amphioxus (Branchiostoma Belcheri Tsingtauense) at different developmental stages. Chin J oceanol limnol, 1997, 15: 236-241

2 Liu Z, Sun Y, Liu N, et al. Characterization, expression, and response to stress of $p 8$ gene in amphioxus. Fish Shellfish Immunol, 2009, 27: $407-413$

3 Kampmeier O F. Evolution and Comparative Morphology of the Lymphatic System. Springfield: Thomas, 1969. 160-180

4 Zhang H W, Huang Z, Yamaguchi K, et al. Granulocytes and macrophages in amphioxus. Zool Sci, 1992, 9: 113-118

5 Cannon J P, Haire R N, Litman G W. Identification of diversified genes that contain immunoglobulin-like variable regions in a protochordate. Nat Immunol, 2002, 3: 1200-1207

6 Dishaw L J, Ota T, Mueller M G, et al. The basis for haplotype complexity in VCBPs, an immune-type receptor in amphioxus. Immunogenetics, 2010, 62: 623-631

7 Han Y, Huang G, Zhang Q, et al. The primitive immune system of amphioxus provides insights into the ancestral structure of the vertebrate immune system. Dev Comp Immunol, 2010, 34: 791-796

8 Lin Y, Chen D, Fan Q, et al. Characterization of SoxB2 and SoxC genes in amphioxus (Branchiostoma belcheri): implications for their evolutionary conservation. Sci China Ser C-Life Sci, 2009, 52: $813-822$
9 Pang Q, Zhang S, Zhao B. Immune parameters in the humoral fluids of amphioxus Branchiostoma belcheri challenged with Vibrio alginolyticus. Fish Shellfish Immunol, 2010, 28: 232-234

10 Yuan S, Liu T, Huang S, et al. Genomic and functional uniqueness of the TNF receptor-associated factor gene family in amphioxus, the basal chordate. J Immunol, 2009, 183: 4560-4568

11 van de Wetering M, Oosterwegel M, van Norren K, et al. Sox-4, an Sry-like HMG box protein, is a transcriptional activator in lymphocytes. EMBO J, 1993, 12: 3847-3854

12 Bogdan C. Nitric oxide and the immune response. Nat Immunol, 2001, 2: 907-916

13 MacAllister R J, Parry H, Kimoto M, et al. Regulation of nitric oxide synthesis by dimethylarginine dimethylaminohydrolase. Br J Pharmacol, 1996, 119: 1533-1540

14 Wilcken D E, Sim A S, Wang J, et al. Asymmetric dimethylarginine (ADMA) in vascular, renal and hepatic disease and the regulatory role of L-arginine on its metabolism. Mol Genet Metab, 2007, 91: 309-317

15 Tung T C, Wu S C, Tung Y F. The development of isolated blastomeres of Amphioxus. Sci Sin, 1958, 7: 1280-1319

16 Yuan S, Huang S, Zhang W, et al. An amphioxus TLR with dynamic embryonic expression pattern responses to pathogens and activates NF-kappaB pathway via MyD88. Mol Immunol, 2009, 46: 2348-2356

17 Chen D, Lin Y, Zhang H. Characterization and expression of two amphioxus DDAH genes originating from an amphioxus-specific gene duplication. Gene, 2008, 410: 75-81

18 Bevins C L, Zasloff M. Peptides from frog skin. Annu Rev Biochem, 1990, 59: 395-414

19 Kollisch G, Kalali B N, Voelcker V, et al. Various members of the Toll-like receptor family contribute to the innate immune response of human epidermal keratinocytes. Immunology, 2005, 114: 531-541

20 Majewska M, Szczepanik M. The role of Toll-like receptors (TLR) in innate and adaptive immune responses and their function in immune response regulation. Postepy Hig Med Dosw (Online), 2006, 60: $52-63$

21 Palm F, Onozato M L, Luo Z, et al. Dimethylarginine dimethylaminohydrolase (DDAH): expression, regulation, and function in the cardiovascular and renal systems. Am J Physiol Heart Circ Physiol, 2007, 293: 3227-3245

22 Huang G, Xie X, Han Y, et al. The identification of lymphocyte-like cells and lymphoid-related genes in amphioxus indicates the twilight for the emergence of adaptive immune system. PLoS ONE, 2007, 2: e206

23 Huang X, Zhang W, Zhang H. Phylogenetic analysis and developmental expression of thymosin-beta4 gene in amphioxus. Dev Genes Evol, 2005, 215: 364-368

Open Access This article is distributed under the terms of the Creative Commons Attribution License which permits any use, distribution, and reproduction in any medium, provided the original author(s) and source are credited. 